\title{
Design of a scanning tunneling microscope for electrochemical applications
}

\author{
Moris M. Dovek, ${ }^{a)}$ Michael J. Heben, ${ }^{\text {b) Christoph A. Lang, }}$ a) \\ Nathan S. Lewis, ${ }^{b, c)}$ and Calvin F. Quate ${ }^{\text {a),c) }}$ \\ Stanford University, Stanford, California 94305 \\ (Received 9 May 1988; accepted for publication 18 July 1988)
}

\begin{abstract}
A design for a scanning tunneling microscope that is well suited for electrochemical investigations is presented. The construction of the microscope ensures that only the tunneling tip and the sample participate in electrochemical reactions. The design also allows rapid replacement of the tip or sample, and enabies facile introduction of auxiliary electrodes for use in electrochemical experiments. The microscope utilizes stepper motor driven approach mechanics in order to achieve fully remote operation and to allow reproducible coarse control of tip/sample spacings for electrochemical experiments. Highly ordered pyrolytic graphite images at atomic resolution in air and aqueous solutions can be obtained with this microscope.
\end{abstract}

\section{INTRODUCTION}

Unlike most other electron- and ion-based surface characterization techniques, scanning tunneling microscopy (STM) has the potential to yieid in-situ information regarding surface structure at liquid interfaces. ${ }^{1}$ For nonreactive surfaces, it has been shown that images obtained with the tunneling microscope are virtually identical for surfaces in contact with air, ${ }^{2,3}$ UHV, ${ }^{4}$ or liquids. ${ }^{5-7}$ Thus the STM is an interesting tool for a number of potential electrochemical applications, including topographic and spectroscopic studies of solid/iquid interfaces, and extremely high resolution lithography., ${ }^{6,8}$ Aditionally, the STM's capability to control tip/sample spacings should allow space-and time-resolved studies of diffusion layer profiles. However, the use of STM in most electrochemical experiments will require modification of conventional gas phase/vacuum microscopes. Important instrumental capabilities include accurate coarse and fine disfance control, remote microscope operation, compatibility with most electrochemical solvents, and the ability to perform conventional potentiostatic and galvanostatic experiments using either the sample or the tip as the working electrode. In this article, we describe a microscope design that has been constructed specifically to achieve these goals and to provide versatile use in electrochemical studies.

There have been a few previous reports of STMs for certain electrochemical experiments. Ex-situ topographical information about surfaces subjected to prior electrochemical cycling, ${ }^{9}$ or passivation ${ }^{11}$ and in-situ images of solutioncovered samples have been obtained. ${ }^{5,7,12,13}$ Also, STM tips have been used as electrodes to electroplate and then image metal deposits on a $220 \mathrm{~nm}$ scale. ${ }^{14}$ The STMs used in these experiments were of varied experimental capability. For example, the design of Sonnenfelo et al. ${ }^{15}$ uses only a drop of liquid, and is, therefore, difficult to operate with high vapor pressure solvents (e.g., $\mathrm{CH}_{3} \mathrm{CN}$ or alcohols) or with auxiliary electrodes, as would be required for most electrochemical investigations. Another microscope designed by Sonnenfeld et $a l^{5}$ is better suited for electrochemical work, but exposes potentially active materials (e.g., steel) to the electrochemical environment. Itaya et $a l^{7}$ have also constructed a tunneling microscope that has produced atomic resolution images in liquids, but their microscope does not allow for reproducible or automated control over large distance excursions in the $z$ direction.

We present a scanning tunneling microscope (STM) that overcomes many of these problems, and also yields atomic resolution images while in an operating electrochemical cell. Noteworthy features of the instrument include a modular design to accommodate rapid tip and/or sample exchange, inert components (e.g., Pyrex, Tefion, etc.) contacting the electrolyte, a large fluid capacity, a novel vibration isolation system, and stepper motor driven approach mechanics. Details of the microscope, as well as examples of its imaging capabilities, are given in the following.

\section{DESIGN OF THE MICROSCOPE}

The structure of the microscope is shown in Fig. 1. The microscope body consists of two major parts: the microscope base and the approach mechanics. These are discussed separately.

\section{A. The base of the microscope}

In order to ensure compatibility with most common electrochemical solvents, the base of the tumneling microscope was made of Pyrex. This base contained mating points for mounting the sample holders, and also provided attachment joints and support for the approach mechanics. Positioning points for the approach mechanics were obtained by grinding three equally spaced dimples into the top surface of the base.

In order to immerse the tip/sample assembly, the microscope required approximately $200 \mathrm{~m} \ell$ of solution; however, straightforward modifications to the microscope would permit use of smaller liquid volumes. A viewing hole was provided in the microscope base in order to facilitate coarse tip/sample positioning in the absence of solution and to allow the inside of the structure to be readily filled with liquid.

The base was mated with a cylindrical Teflon support that was machined to fit snuggly into, and lie at the bottom 

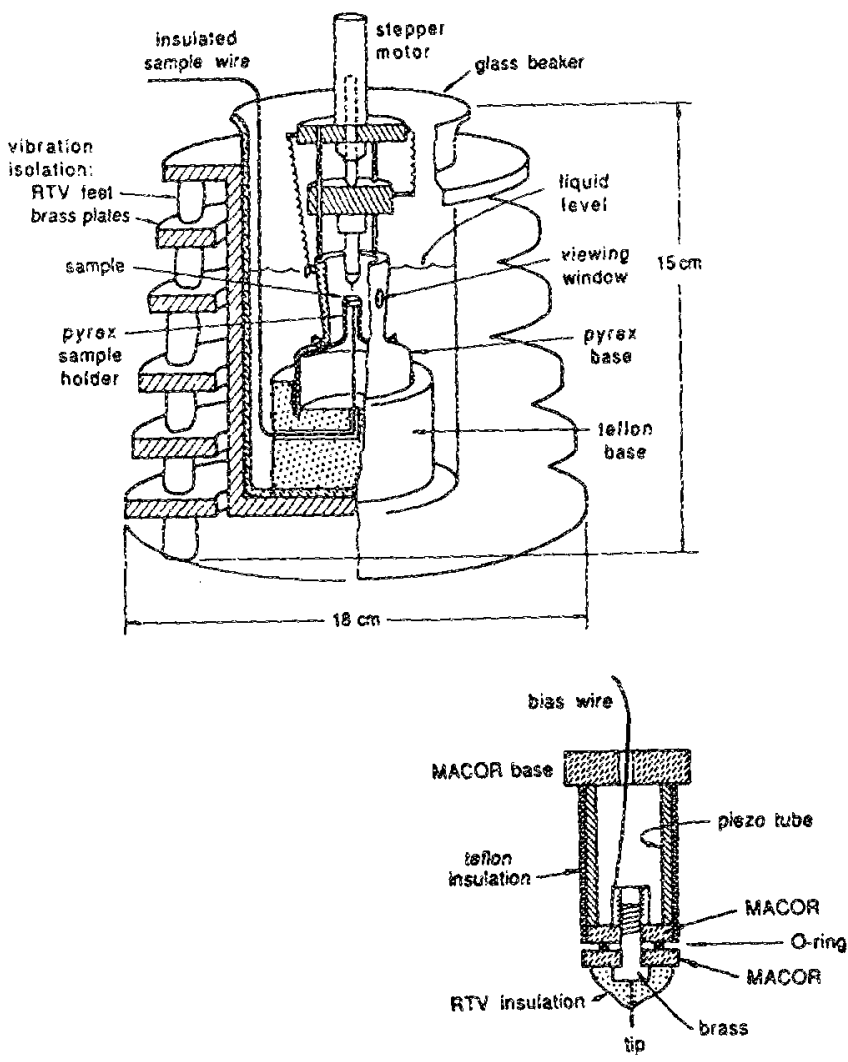

FIG. 1. Idealized depiction of the scanning tunneling microscope designed for electrochemical applications. The inset shows a detailed view of the scanner construction.

of, the solution containment vessel. This Tefon support served to occupy some of the volume within the beaker and thereby reduce the amount of liquid needed to completely cover the tip and sample. More importantly, the weight of the Teflon greatly enhanced the stability of the microscope by lowering its center of mass. This resulted in substantial reductions in the effectiveness of vibrational coupling to the STM.

The vibration isolation system employed in the microscope was similar to an arrangement described by Binnig et $a l .{ }^{16}$ It consisted of brass plates separated by silicone rubber feet (see Fig. 1). This arrangement has been shown to yield excellent isolation with minimum space and machining requirements.

\section{B. The approach mechanics}

The second major part of the STM is the approach mechanies for the tip. In our design, the mechanics were held in position on the Pyrex base by the use of springs. As indicated in Fig. 1, the spring tension was applied between attachment hooks that were part of the base and small bolts that protruded from the uppermost stainless-steel plate of the approach mechanics.

Guidance rails for the piezoelectric scanner and the tip were fabricated from three stainless-steel rods. The rods were press-ift in a steel plate, and also were mated with the dimples in the Pyrex base. This arrangement provided a method to align the upper and lower portions of the microscope. The scanner and tip were mounted on a second, lower stainless-steel plate. This plate was spring loaded to the upper plate by two springs. This arrangement was used to achieve an asymmetric loading for improved stability in the plane of the sample. To obtain a controlled approach between the sample and the tip, the second plate was pushed toward the sample by a stepper motor ${ }^{17}$ that was anchored to the upper plate.

\section{Sample hoiders}

Sample holders were made of $P_{y}$ rex, and were shaped in the form of a disk with a centered mesa. The holders had a capillary opening along the axis of the mesa and through the center of the disk. The sample holders were affixed to the base of the microscope using Tefion screws, which were directed through two slots that were machined in the sample holder disk.

Samples were cemented into the top of the mesa with silver paint, and electrical connections were made via the capillary opening. After mouning, the edges of the sample and the Ag paint were insulated from solution with epoxy. The sample wire was fully insulated from the solution by Tefon tubing, and the wire traveled to a current preamplifier that was positioned just outside of the solution.

\section{The piezoelectric scanner and tip mounting}

The single tube piezo was constructed from $\mathrm{PZT}-5 \mathrm{H}_{9}{ }^{18}$ and was of the design described by Binnig and Smith. ${ }^{19}$ The piezo tube was protected from solution by a sheath made of heat-shrink-type Teflon tubing and mounted on a MACOR block.

To facilitate mounting of tip holders, a piece of brass with female threads was cemented into the piezo tube (Fig. 1 inset). Threaded tip holders were screwed into the brass piece to form an O-ring compression seal with the piezo. Additionally, a drop of silicone rubber sealant was used to insulate the exposed metal of the uncoated tip shaft and mounting chuck. This procedure enabled rapid interchange of tips, and also allowed mounting of several alternate tips without disturbing the operation of the microscope.

\section{E. Stepper motor drive electronics}

Circuitry was constructed in order to drive the stepper motor with either pulsed or de voltages. Under dc operation, the tip could be translated at rates up to $0.2 \mathrm{~mm} / \mathrm{s}$ either toward or away from the sample. This capability was found to be extremely useful in coarse tip/sample positioning. In the pulse mode, the stepper mode could be driven either with a train of pulses or with a single pulse. The pulses were of 10$\mathrm{ms}$ duration and consisted of arbitrarily programmable voltages between the limits of 3.5 to $6 \mathrm{~V}$.

The amount of tip travel in response to a single voltage puise was found to be a function of the stiffness of the springs employed to load the stepper motor. By appropriate adjustment of loading and pulse voltage, it was found that the steel plate that carried the piezo and tip could be reproducibly 
moved as little as $100 \AA$ in response to a single, manual voltage pulse to the stepper motor.

In automatic mode, the circuitry sent 10 -ms pulses to the stepper motor every $100 \mathrm{~ms}$. This mode was extremely useful for the final approach of the tip into tunneling range. To prevent shorting between the tip and the sample, the pulse rrain was terminated if a current in excess of a given setpoint value (typically $1 \mathrm{nA}$ ) was sensed. Exceeding the setpoint also caused the circuitry to fully retract the piezo tube by applying the full voltage (approximately $150 \mathrm{~V}$ ) to the piezo. Tunneling was then reesiablished by allowing the piezo voltage to capacitively discharge through a $R C$ circuit, which enabled a smooth approach of the tip toward the sampie until feedback control was established.

\section{I!. DISCUSSION}

As just described, the overall construction and choice of materials make this microscope modular and chemically inert. In particular, the threaded tip holders and slotted sample holders enable rapid tip/sample exchange, while all metallic or potentially reactive surfaces are insulated from solution by either Teflon, chemically inert epoxy, or neoprene. Furthermore, the large fluid capacity of the STM avoids solution compositional changes and greatly facilitates inclusion of auxiliary electrodes for electrochemical investigations. This combination of properties makes the instrument quite attractive for use in electrochemical applications.

The use of a stepper motor to move the scanner and tip made this STM somewhat top heavy and, therefore, led to increased susceptibility to vibrations. To counteract this, the Teflon support (which serves to lower the center of mass of the microscope itself) and the brass vibration isolation plates were designed to work in concert to effectively isolate the STM from low-frequency mechanical vibrations. Furthermore, when loaded with the microscope and solution, the system of plates served to lower the center of gravity of the whole system (Fig. 1). Additionally, the lossy silicone rubber feet proved to provide effective damping between adjacent plates. The entire system was found to provide sufficient stability that atomic resolution images of graphite and $\mathrm{MoS}_{2}$ could be obtained under a variety of different conditions.

An advantageous feature of this microscope design is that the stepper motor driven approach mechanics and supporting electronics provide capabilities for fully remote operation. This automatic approach facility, in conjunction with the modularity of the microscope design, make this instrument ideal for use in inert atmosphere gloveboxes where manual dexterity is compromised. Because tip speeds in the automatic approach mode range from 0.1 to $5 \mu \mathrm{m} / \mathrm{s}$ (depending on spring tension and voltage pulse height), macroscopically large distances (ca. $100 \mu \mathrm{m}$ ) can be traversed in less than 1 min. Thus, the conditions on coarse tip/sample positioning are less stringent than with manual controls, where the tunneling tip must be visually placed within a few microns of the sample for the rapid final approach into tunneling range. This arrangement also minimized inadvertent "crashing" of the tip (direct physical contact between the tip and the sample) during coarse positioning. In fact, it was found that the ability to detect tunneling current, deactivate

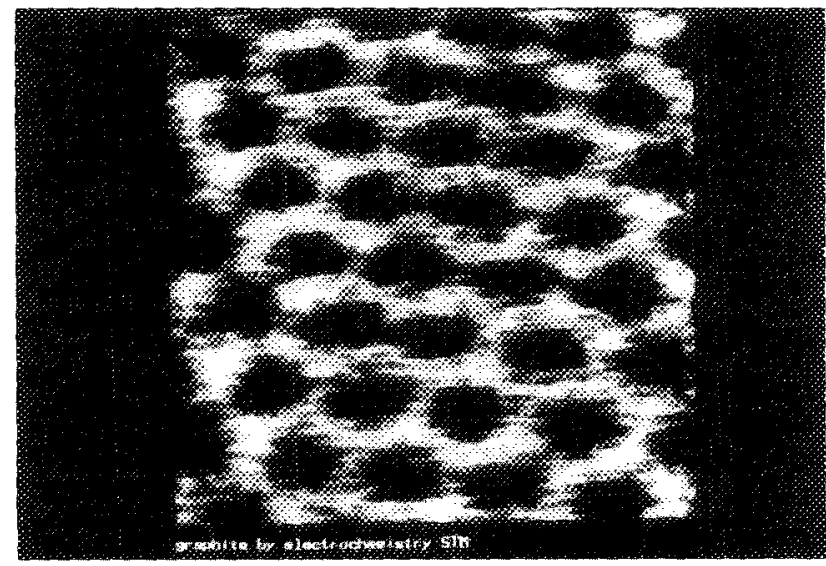

Fig. 2. Inage of highly ordered pyrolytic graphite taken under $1 \mathrm{M} \mathrm{NaCl}$ with our STM. The tip bias was $+550 \mathrm{mV}$ and the tunneling current was 1 nA. Commercially available glass-coated Pt-Ir tips"' were usch. In this representation dark spots correspond to carbon aloms at a separation of $2.5 \AA$.

the pulse train, and retract and relax the piezo tube were so effective at preventing tip/sample shorting that most $W$ and Pt-Ir tips directly yielded atomic resolution images.

Figure 2 is an image of highly ordered pyrolytic graphite obtained under an aqueous 1-M NaCl solution with our microscope operating in fast scan mode. ${ }^{20}$ It is virtually identical to images we have obtained of these surfaces in contact with air. These images compare quite favorably with those of pyrolytic graphite obtained by Schneir and Hansma (in nonpolar liquids), Itaya and Sugawara (in DI water), and Sonnenfeld and Schardt ${ }^{12}$ (in $0.050 \mathrm{M} \mathrm{AgClO}_{4}$ ). Of note is that all of these cases, including our own, required the use of glass-coated Pt-Ir tips ${ }^{21}$ that limit the amount of exposed metal area in order to minimize residual Faradaic currents.

A final noteworthy feature of the microscope is the ability to accurately achieve large variations in the distance between the tip and sample. To achive such control, single voltage puises are employed to adjust the 2 -piezo voltage at which tunneling occurs. Complete retraction of the piezo then yields the desired tip/sample spacing. This technique can be used to accurately control tip/sample distances for values as large as the full range of the piezo tube $(0.75 \mu \mathrm{m})$. Calibration of the piezo travel in response to a pulse (for a given pulse height and set of springs) allows coarser distance variation over the travel of the stepper motor. This procedure has applications in potentiometric, galvanostatic, or cyclic voltametry experiments where the STM tip is used as a microelectrode at well-defined small tip/sample spacings. We are currently utilizing these instrumental capabilities in several electroanalytical applications, and will describe the results of these experiments in subsequent publications.

In conclusion, we have constructed a scanning tunneling microscope that is well suited for electrochemical investigations. This STM incorporates several novel features that have not been employed elsewhere, including remote operation, compatibility with electrochemical solvents, variable 
distance control, facile tip/sample interchange, modular design, and adaptability to most electroanalytical applications. With this microscope, highly ordered pyrolytic graphite has been imaged at atomic resolution in air and in aqueous solutions.

\section{ACKNOWLEDGMENTS}

The authors would like to thank Alison Baski, Jun Nogami, and Reggie Penner for useful discussions and help with construction of the microscope. We acknowledge the support of an IBM Predoctoral Manufacturing Fellowship (M. M. D.) and a German National Scholarship Foundation Overseas Fellowship (C. A. L.). N. S. L. acknowledges support as a Dreyfus Teacher-Scholar and as an A. P. Sloan Fellow. N. S. L. also acknowledges financial assistance from the Office of Naval Research, Grant No. No0014-85-K0805. C.F. Q. acknowledges support of the Defense Advanced Research Projects Agency.

\footnotetext{
${ }^{a)}$ Department of Appiled Physics.

b) Department of Chemistry.

c) Address correspondence to these authors.

'M. M. Dovek, M.J. Heben, N.S. Lewis, R. M. Penner, and C. F. Quate, in

"Molecular Phenomena at Electrode Surfaces," ACS Symposia Series, edited by M. P. Soriaga (in press).
}

${ }^{5}$ S. Park and C. F. Quate, Appl. Phys. Lett. 48, 112 (1986).

3. Schneir, R. Sonnenfeld, P. K. Hansma, and J. Tersoff, Phys. Rev. B 34, $4979(1986)$.

${ }^{+}$G. Binnig, H. Fuchs, Ch. Gerber, H. Rohrer, E. Stoll, and E. Tosatti, Europhys. Lett. 1, 31 (1986).

${ }^{5}$ R. Sonnenfeld and P. K. Hansma, Science 232, 211 (1986).

"J. Schneir and P. K. Hansma, Langmuir 3, 1025 (1986).

${ }^{7}$ K. Itaya and S. Sugawara, Chem. Lett. 10, 1927 (1987).

${ }^{8} \mathrm{C}$. W. Lin, F. F. Fan, and A. 3. Bard, J. Ejectrochem. Soc. 134, 1038 (1987).

"L. Vasquez, J. Gomez, A. M. Baro, N. Garcia, M. L. Marcos, J. Gonzalez Velasco, J. M. Vara, A. J. Arvia, J. Presa, A. Garcia, and M. Aguilar, I. Am. Chem. Soc. 109, 1730 (1987).

"F.F. Fan and A. J. Bard, Anal. Chem. 60, 751 (1988).

${ }^{11}$ S. Morita, K. Itaya, and N. Mikoshiba, Jpn. J. Appl. Phys. 25, L743 (1986).

${ }^{12}$ R. Sonnenfeld and B. C. Schardt, Appl. Phys. Lett. 49, 1172 (1986).

${ }^{13}$ K. Iraya, K. Higaki, and S. Sugawara, Chem. Iett. 3, 421 (1988).

${ }^{14}$ J. Schneir, P. K. Hansma, V. Elings, J. Gurley, K. Wickramasinghe, and R. Sonnenfeld, SPIE'88 Conference Proceedings (in press).

${ }^{15}$ R. Sonnenfeld, J. Schneir, H. Drake, P. K. Hansma, and D. L. Aspnes, Appl. Phys. Lett. 50, 1742 (1987).

"G. Binnig, C. Gerber, and O. Marti, IBM Tech. Discl. Bull. 27, 3137 (1984).

17 From Newport Corporation, model $\$ 860-05$.

"Available from E.B.L. Co., 91 Prestige Circle, East Hartford, CT 06108.

"G. Binnig and D. P. E. Smith, Rev. Sci. Instrum. 57, 1688 (1986).

${ }^{26}$ A. Bryant, D. P. E. Smith, and C. F. Quate, Appl. Phys. Lett. 48, 832 (1986).

${ }^{2} 0.020$ " Pt-Ir (30\%) avallable from Longreach Scientific Resources, RFD 1, Box 549, Orr's Island, ME 04066. 